www.Volsu.ru

DOI: https://doi.org/10.15688/jvolsu3.2017.4.12

UDC $330.45,519.865 .2$

LBC 65.011, 65в $6,22.18$

\title{
BENEFIT'S DIVISION WITH TWO INDIVISIBLE COMPONENTS BY THE PROCEDURE ALLOWING NEGOTIATIONS
}

\author{
Vladislav V. Egorov \\ Volgograd State University, Volgograd, Russian Federation
}

\begin{abstract}
The article deals with the distribution of partially divisible benefit with two indivisible components between the two beneficiaries at the independent informing each other about their discrete utility functions. The specified information represents the way of division desirable to the beneficiary and the maximum price which they are ready to pay for the implementation of the proposed approach to the benefit's distribution. The study of the problem is carried out with the assumption of equality of rights and opportunities for beneficiaries which is possible by attracting an arbitrator, independent and indifferent to the participants in the conflict process. The author describes types and conditions of the conflicts' emergence between the beneficiaries connected with the lack of consensus both on the way of division and on the amount of compensation payment. The article presents the classification of conflicts into one-type groups. The author proposes the optimal ways of division and specially calculated compensation values, not taking into account the factor of envy, understood as the situation when one of the beneficiaries is sure that he/she gets less than the competitor. The cases when it is possible to do without negotiations on the division issue are listed. The author reveals the circumstances when negotiations are possible. Conditions of inevitability of negotiations are pointed out. The cross-disciplinary research is carried out on a joint of mathematics and the social and economic sphere, with the use of their knowledge, provisions, methods. The research results can be used in practice in economic activity and political field for smoothing contradictions, typical for human society.

Key words: resources distribution, partially divisible benefit, division of inheritance, conflict, conciliation of interests, negotiations.
\end{abstract}

УДК $330.45,519.865 .2$

ББК $65.011,65 \mathrm{~B} 6,22.18$

\section{К ВОПРОСУ ДЕЛЕЖА БЛАГА \\ С ДВУМЯ НЕДЕЛИМЫМИ СОСТАВЛЯЮЩИМИ ПОСРЕДСТВОМ ПРОЦЕДУРЫ, ДОПУСКАЮЩЕЙ ПЕРЕГОВОРЫ}

\author{
Владислав Валерьевич Егоров \\ Волгоградский государственный университет, г. Волгоград, Российская Федерация
}

\begin{abstract}
Аннотация. Рассмотрено распределение частично делимого блага с двумя неделимыми составляющими между двумя благополучателями при независимом сообщении ими друг другу неполной информации о своих дискретных функциях полезности. Указанная информация представляет собой желаемый бенефициаром способ дележа и максимальную цену, которую он готов заплатить за реализацию предлагаемого им подхода к распределению блага. Изучение проблемы осуществлено при предположении равенства прав и возможностей благополучателей, учет которого возможен посредством привлечения арбитра, независимого и индифферен공 тного по отношению к участникам конфликтного процесса. Представлены типы и условия возникновения ضें конфликтов интересов между благополучателями, как связанные с отсутствием согласованности мнений о способе дележа, так и с отсутствием согласованности мнений о величине его оплаты в виде компенсаций. : П․ Предложена классификация всевозможных случаев с разбиением их на однотипные группы. При отсутствии 空 учета «зависти», понимаемой как ситуация, когда кто-то из благополучателей считает, что ему достается мень(2) ше, чем конкуренту, предложены удовлетворяющие благополучателей способы дележа и рассчитанные специ-
\end{abstract}


альным образом значения компенсаций. Перечислены случаи, когда без переговоров по вопросам дележа можно обойтись, а значит допустим формальный подход к решению спорной ситуации. Выявлены обстоятельства, когда переговоры возможны. Найдены условия, при которых в указанных обстоятельствах переговоров не избежать. Исследование выполнено на стыке математики и социально-экономической сферы, использует их знания, положения и методы, а его результаты могут быть использованы на практике в хозяйственной деятельности и политической области для сглаживания типичных для человеческого общества противоречий.

Ключевые слова: распределение ресурсов, частично делимое благо, дележ наследства, конфликт, согласование интересов, переговоры.

\section{Введение}

В практике экономических субъектов неизменно появляются обстоятельства, приводящие к необходимости производить распределение имеющихся ресурсов, в том числе достающихся им не только в результате их хозяйственной практики. Доступ к тем или иным благам также может возникать в результате делегирования или передачи прав на их владение и использование из-за нежелания или невозможности прежнего владельца осуществлять далее подобную деятельность самостоятельно. Этот доступ может быть получен и в результате различных открытий в ходе научно-технического прогресса и т. д. В случае, когда на предоставленное или вновь образованное благо претендуют несколько благополучателей, необходимо искать новые решения хозяйственных, наследственных, территориальных и прочих споров, если в законах, регламентирующих жизнь общества (от межличностного уровня до международного), ранее не были прописаны соответствующие указания.

Не затрагивая известную социально-экономическую дилемму «справедливость-эффективность», в настоящей работе предлагаются решения лишь с точки зрения императива равенства сторон спора или конфликта.

Многие вопросы дележа хорошо изучены и связаны с актуальными социально-экономическими и политическими аспектами деятельности человеческих сообществ $[1 ; 5]$. Однако существует целый класс недостаточно изученных проблем, в частности, относящихся к ситуациям дележа дискретных благ, не являющихся бесконечно делимыми. Но если в отношении этого в работах $[2 ; 3 ; 4]$ исследуется процедура дележа, предполагающая, что благополучатели независимо друг от друга один раз и сразу сообщают друг другу свои системы индивидуальных предпочтений полностью, то, поскольку возможна и иная организация процесса дележа, имеет смысл рассмотреть случаи частичной информированности о данных системах. Разумно предположить, что это может вызвать необходимость вести уже не разовые, а потенциально допустимые многоэтапные переговоры, математическая теория которых, изложенная в [5], не касается тематики данной статьи.

Сформулируем и конкретизируем проблему, связанную с согласованием позиций по поводу дележа имеющегося ресурса.

\section{Основные положения и постановка задачи}

Пусть частично делимое благо, ресурс или наследство складывается из двух неделимых составляющих его частей. Рассмотрим дележ этого блага между двумя конкурентами-благополучателями или, можно говорить, наследниками-бенефициарами $H^{1}$ и $H^{2}$.

При этом изучение учета «зависти», понимаемой в смысле получения наследниками разных относительных значений добавок сверх тех денежных эквивалентов блага, на которые они рассчитывали в худшем для себя случае, оставим для дальнейшего исследования.

Обозначим возможные исходы дележа в виде: $\left(\delta_{1}, \delta_{2}\right)$, где $\delta_{i}=\left\{\begin{array}{ll}1, & W_{i} \in H^{1} \\ 0, & W_{i} \notin H^{1}\end{array}\right.$. Тогда всевозможные способы дележа таковы: $(0,0)$, $(0,1),(1,0),(1,1)$. Например, исход $(1,0)$ означает что подблаго $W_{1}$ предлагается отдать наследнику $H^{1}$, а подблаго $W_{2}$ предлагается ему не отдавать и, следовательно, оно должно достаться наследнику $H^{2}$ (то есть указанное обозначение однозначно определяет, что должно достаться наследнику $H^{2}$ в случае реализации представленного исхода). 
Пусть каждый наследник $H^{z}$ независимо от другого наследника $H^{\bar{z}}$ (где $\bar{z}$ означает «не $z$ ») предлагает свой вариант исхода дележа в виде $\left(\delta_{1}, \delta_{2}\right)$ и сообщает, какую максимальную сумму $\bigsqcup_{\delta_{1} \delta_{2}}^{z} / 2=\bigsqcup^{z}\left(\delta_{1}, \delta_{2}\right) / 2$ условных денежных единиц (у.д.е.) он готов заплатить конкуренту за реализацию такого исхода. Иными словами, таким образом наследник дает понять, что этот результат дележа он оценивает не более, чем в $Ц_{\delta, \delta}^{z}$ у.д.е. и не более, чем половину из которых он готов отдать конкуренту.

Так, например, если наследник $H^{1}$ предлагает дележ вида $(1,0)$ и сообщает, что готов заплатить за него максимум $\bigsqcup_{10}^{1} / 2=100$ у.д.е., то фактически это означает, что с его точки зрения цена подблага $W_{1}$ (используемого без подблага $W_{2}$ ) составляет максимум 200 у.д.е.

При этом вполне возможно наличие следующих однотипных ситуаций: $Ц_{01}^{1} / 2>Ц_{11}^{1} / 2$ или $\bigsqcup_{01}^{2} / 2>\bigsqcup_{00}^{2} / 2$ и т. д. Они означают, что соответствующему наследнику, согласно его дискретной функции полезности, важнее получить некоторую часть блага, но не особенно важно получить все благо целиком, например, так как это потребует впоследствии нежелательных расходов на его содержание или из-за особенностей имеющейся у наследника системы интересов.

Заметим, что если за реализацию $(1,1)$ дележа наследник $H^{1}$ готов заплатить $L_{11}^{1} / 2$, то и за соглашение с реализацией $(0,0)$ деле- жа, когда все достается его конкуренту, наследник $H^{1}$ считает необходимым получить от него компенсацию, не меньшую, чем $Ц_{11}^{1} / 2$.

Формально говоря, $\bigsqcup_{00}^{1} / 2=-Ц_{11}^{1} / 2<0$. Аналогично: $\bigsqcup_{11}^{2} / 2=-Ц_{00}^{2} / 2<0$.

Таким образом, рассматривается предельная ситуация. Однако если в дальнейших исследованиях дополнительно учитывать психологический фактор, то следует предусматривать, что, как правило, имеет место некоторое различие между этими ценами, а именно, за возможность получения блага наследник может быть готов платить несколько больше, нежели за отказ от него.

Как при этом будет определяться величина компенсации $K_{\delta_{1} \delta_{2}}^{z}$ от наследника $H^{z}$ к его конкуренту за реализацию дележа в виде $\left(\delta_{1}, \delta_{2}\right)$ описывается далее.

Представим случаи описанной проблемы в виде таблицы всех возможных для предложения наследниками способов дележей и их результатов (см. таблицу). Например, когда наследник $H^{1}$ выдвигает вариант дележа $(1,0)$ (сообщая цену $Ц_{10}^{1} / 2$ ), а наследник $H^{2}$ выдвигает вариант дележа $(0,0)$ (сообщая цену $\left.L_{00}^{2} / 2\right)$, то в таблице это выглядит как «Случай $4.3 »$, в отношении которого сделан вывод, что в рамках него «переговоры не нужны», согласно рассуждениям, приведенным в статье далее.

Кроме того, если наследники не смогут договориться друг с другом за очередной этап переговоров, на котором каждый из них дела-

Таблица

Типология поведения наследников и соответствующих результатов их взаимодействия

\begin{tabular}{|c|c|c|c|c|c|}
\hline & \multicolumn{4}{|c|}{$H^{-1}$} \\
\hline & & $(0,0)$ & $(0,1)$ & $(1,0)$ & $(1,1)$ \\
\hline & & $\bigsqcup_{00}^{2} / 2$ & $Ц_{01}^{2} / 2$ & $\bigsqcup_{10}^{2} / 2$ & $\begin{array}{c}\bigsqcup_{11}^{2} / 2= \\
=-\bigsqcup_{00}^{2} / 2<0\end{array}$ \\
\hline$(1,1)$ & 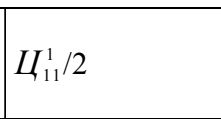 & $\begin{array}{c}\text { Случай } 6 \\
\text { (переговоры } \\
\text { не нужны) }\end{array}$ & $\begin{array}{c}\text { Случай } 4.1 \\
\text { (переговоры } \\
\text { не нужны) }\end{array}$ & $\begin{array}{c}\text { Случай } 4.2 \\
\text { (переговоры } \\
\text { не нужны) }\end{array}$ & $\begin{array}{c}\text { Случай } 1.1 \\
\text { (переговоры } \\
\text { не нужны) }\end{array}$ \\
\hline$(1,0)$ & 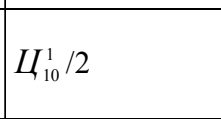 & $\begin{array}{c}\text { Случай } 4.3 \\
\text { (переговоры } \\
\text { не нужны) }\end{array}$ & $\begin{array}{c}\text { Случай } 3.1 \\
\text { (переговоры } \\
\text { не нужны) }\end{array}$ & $\begin{array}{c}\text { Случай } 2.1 \\
\text { (переговоры } \\
\text { не нужны) }\end{array}$ & $\begin{array}{c}\text { Случай } 5.2 \\
\text { (переговоры } \\
\text { возможны) }\end{array}$ \\
\hline$(0,1)$ & $\bigsqcup_{01}^{1} / 2$ & $\begin{array}{c}\text { Случай } 4.4 \\
\text { (переговоры } \\
\text { не нужны) }\end{array}$ & $\begin{array}{c}\text { Случай } 2.2 \\
\text { (переговоры } \\
\text { не нужны) }\end{array}$ & $\begin{array}{c}\text { Случай } 3.2 \\
\text { (переговоры } \\
\text { не нужны) }\end{array}$ & $\begin{array}{c}\text { Случай } 5.1 \\
\text { (переговоры } \\
\text { возможны) }\end{array}$ \\
\hline$(0,0)$ & $\begin{array}{l}Ц_{00}^{1} / 2= \\
=-Ц_{11}^{1} / 2<0\end{array}$ & $\begin{array}{c}\text { Случай } 1.2 \\
\text { (переговоры } \\
\text { не нужны) }\end{array}$ & $\begin{array}{c}\text { Случай } 5.4 \\
\text { (переговоры } \\
\text { возможны) }\end{array}$ & $\begin{array}{c}\text { Случай } 5.3 \\
\text { (переговоры } \\
\text { возможны) }\end{array}$ & $\begin{array}{c}\text { Случай } 7 \\
\text { (переговоры } \\
\text { не нужны) }\end{array}$ \\
\hline
\end{tabular}

Примечание. Составлено автором; пояснения см. ниже. 
ет другому по одному предложению о способе дележа, то проводится следующий этап переговоров и т. д.

При этом до начала всех переговоров, исходя из инфляционных, амортизационных и прочих соображений, определяется коэффициент дисконтирования $d \mathrm{O}(0,1)$, на который после каждого этапа будет умножаться каждая цена из ранее названных наследниками, причем с сохранением ранжирования предпочтений. Имеется в виду, что если на первом этапе, например, наследник $H^{1}$ предложит дележ $(1,0)$ с ценой $\bigsqcup_{10}^{1} / 2$, то для этого наследника все прочие способы дележа, возможные для него, должны считаться не лучше этого, а соответствующие им цены - не больше этой, то есть $\bigsqcup_{10}^{1} \geq \max \left\{Ц_{00}^{1}, Ц_{01}^{1}, \bigsqcup_{11}^{1}\right\}$. А на следующем этапе наследником $H^{1}$ снова может быть предложен любой дележ (в том числе и предлагавшийся ранее), но с ценой не большей $\delta \cdot Ц_{10}^{1} / 2$.

\section{Решение проблемы дележа при неполной информации о системе предпочтений благополучателей}

\section{Случай 1.1. таблицы.}

1.1.1. Так как каждый наследник предлагает одинаковый исход дележа (когда и подблаго $W_{1}$, и подблаго $W_{2}$, то есть все наследство $W_{12}$ целиком предлагается передать наследнику $H^{1}$, который в таком случае должен выплатить наследнику $H^{2}$ некоторую компенсацию), то здесь нет конфликта интересов при $\bigsqcup_{11}^{1} / 2 \geq-\bigsqcup_{11}^{2} / 2$, то есть в случае готовности наследника $H^{1}$ заплатить наследнику $H^{2}$ за реализацию указанного дележа компенсацию, не меньшую, чем тот рассчитывает получить за свой отказ от блага $W_{12}$.

При этом компромиссная или справедливая (в смысле равноправия сторон по отношению к распределяемому между ними благу) компенсация от наследника $H^{1}$ наследнику-конкуренту $H^{2}$ составит половину от указанного типа справедливой цены результата дележа, то есть величину $K_{11}^{1}=\left(\frac{Ц_{11}^{1}+\left(-Ц_{11}^{2}\right)}{2}\right) / 2=\frac{Ц_{11}^{1}+\left(-Ц_{11}^{2}\right)}{4}$ у.д.е.

Реализовав дележ указанным образом, наследники $H^{1}$ и $H^{2}$ будут полагать, что в денежном выражении они получают, соответственно:

$$
\begin{gathered}
D_{11}^{1}=Ц_{11}^{1}-\frac{Ц_{11}^{1}+\left(-Ц_{11}^{2}\right)}{4}=\frac{3 Ц_{11}^{1}+Ц_{11}^{2}}{4} \geq 0 \\
D_{11}^{2}=\frac{Ц_{11}^{1}+\left(-Ц_{11}^{2}\right)}{4} \geq 0 .
\end{gathered}
$$$$
\text { и }
$$

Покажем, что в этом случае каждый наследник получит не меньше, чем им допускалось при предложенном дележе, а значит при отсутствии учета «зависти» он не откажется от такого распределения блага, требуя дополнительных переговоров. Действительно, согласно приведенным здесь неравенствам имеем:

$$
\begin{aligned}
& D_{11}^{1}-\frac{Ц_{11}^{1}}{2}=\frac{3 Ц_{11}^{1}+Ц_{11}^{2}}{4}-\frac{Ц_{11}^{1}}{2}=\frac{Ц_{11}^{1}+Ц_{11}^{2}}{4} \geq 0 \\
& \text { и } D_{11}^{2}-\frac{-Ц_{11}^{2}}{2}=\frac{Ц_{11}^{1}+\left(-Ц_{11}^{2}\right)}{4}-\frac{-Ц_{11}^{2}}{2}=\frac{Ц_{11}^{1}+Ц_{11}^{2}}{4} \geq 0 \text {. }
\end{aligned}
$$

1.1.2. Однако конфликт интересов в отношении оплаты дележа будет иметь место при $\bigsqcup_{11}^{1} / 2<-\bigsqcup_{11}^{2} / 2$ (то есть в этом случае за свой отказ от блага $W_{12}$ наследник $H^{2}$ не сможет получить от наследника $H^{1}$ желаемые - $\bigsqcup_{11}^{2} / 2$ у.д.е., поскольку тот готов заплатить максимум $\bigsqcup_{11}^{1} / 2$ у.д.е.).

Но поскольку $\bigsqcup_{11}^{2} / 2=-\bigsqcup_{00}^{2} / 2$, следует реализовать дележ $(0,0)$, отдав все благо $W_{12}$ наследнику $H^{2}$, который должен будет заплатить наследнику $H^{1}$ компромиссную или справедливую в указанном ранее смысле компенсацию $K_{00}^{2}=\left(\frac{\bigsqcup_{11}^{1}+\left(-Ц_{11}^{2}\right)}{2}\right) / 2=\frac{Ц_{11}^{1}+\left(-Ц_{11}^{2}\right)}{4}$ у.д.е.

И тогда наследники $H^{1}$ и $H^{2}$ будут полагать, что в денежном выражении они получают, соответственно:

$$
D_{11}^{1}=D_{11}^{1}(0,0)=\frac{Ц_{11}^{1}+\left(-Ц_{11}^{2}\right)}{4} \geq 0
$$

и $D_{11}^{1}=\left(-Ц_{11}^{2}\right)-\frac{Ц_{11}^{1}+\left(-Ц_{11}^{2}\right)}{4}=\frac{-Ц_{11}^{1}-3 Ц_{11}^{2}}{4} \geq 0$.

Здесь запись $D_{11}^{1}=D_{11}^{1}(0,0)$ - это дополнительное более полное указание на то, что рассматривается денежное выражение получаемого наследником $H^{2}$ при предложенном им дележе $(1,1)$, а реализованном $(0,0)$.

И в этом случае, как и в п. 1.1.1., доказывается, что каждый наследник получит не меньше, чем им допускалось при предложенном дележе, а значит при отсутствии учета «зависти» не откажется от обсуждаемого распределения блага, требуя дополнительных переговоров. 
Случай 1.2. таблицы аналогичен случаю 1.1. Тогда:

1.2.1. Здесь нет конфликта интересов при $Ц_{00}^{2} / 2 \geq-Ц_{00}^{1} / 2$ (когда и подблаго $W_{1}$, и подблаго $W_{2}$, то есть все наследство $W_{12}$ целиком предлагается передать наследнику $H^{2}$, который в таком случае должен выплатить наследнику $H^{1}$ некоторую компенсацию и, согласно приведенному неравенству, готов это сделать). При этом величина компенсации будет равна:

$$
K_{00}^{2}=\left(\frac{-Ц_{00}^{1}+Ц_{00}^{2}}{2}\right) / 2=\frac{-Ц_{00}^{1}+Ц_{00}^{2}}{4} \text { у.д.е. }
$$

А реализовав дележ указанным образом, наследники $H^{1}$ и $H^{2}$ будут полагать, что в денежном выражении они получают, соответственно:

$$
\begin{gathered}
D_{00}^{1}=\frac{-Ц_{00}^{1}+Ц_{00}^{2}}{4} \geq 0 \\
\text { и } \quad D_{00}^{2}=Ц_{00}^{2}-\frac{-Ц_{00}^{1}+Ц_{00}^{2}}{4}=\frac{Ц_{00}^{1}+3 Ц_{00}^{2}}{4} \geq 0 .
\end{gathered}
$$

1.2.2. Здесь есть конфликт интересов в отношении оплаты дележа при $\bigsqcup_{00}^{2} / 2<-\bigsqcup_{00}^{1} / 2$ (когда за свой отказ от блага $W_{12}$ наследник $H^{1}$ не сможет получить от наследника $H^{2}$ желаемые $-\bigsqcup_{00}^{1} / 2$ у.д.е., поскольку тот готов заплатить максимум $\bigsqcup_{00}^{2} / 2$ у.д.е.). Тогда производится дележ в виде $(1,1)$ с выплатой компенсации $K_{00}^{2}=\frac{-Ц_{00}^{1}+Ц_{00}^{2}}{4}$ у.д.е. от наследника $H^{1}$ наследнику $H^{2}$, которые будут полагать, что в денежном выражении они получают, соответственно:

и

$$
\begin{gathered}
D_{00}^{1}=D_{00}^{1}(1,1)=\frac{-3 Ц_{00}^{1}+Ц_{00}^{2}}{4} \geq 0 \\
D_{00}^{2}=\frac{-Ц_{00}^{1}+Ц_{00}^{2}}{4} \geq 0 .
\end{gathered}
$$

\section{Случай 2.1. таблицы.}

Здесь нет конфликта интересов в отношении способа дележа, так как каждый наследник предлагает одинаковый исход дележа, а именно: подблаго $W_{1}$ достается наследнику $H^{1}$, и он готов платить за это не больше $\bigsqcup_{10}^{1} / 2$ наследнику $H^{2}$, если тот будет против. А подблаго $W_{2}$ достается наследнику $H^{2}$, и он готов платить за это не больше $\bigsqcup_{10}^{2} / 2$ наслед- нику $H^{1}$, если тот будет против. Но поскольку никто не против, то и платить какие-либо компенсации никто своему конкуренту не должен.

Тогда, реализовав дележ указанным образом, наследники $H^{1}$ и $H^{2}$ будут полагать, что в денежном выражении они получают, соответственно: $D_{10}^{1}=\bigsqcup_{10}^{1} / 2$ у.д.е. и $D_{10}^{2}=\bigsqcup_{10}^{2} / 2$ у.д.е., то есть не меньше, чем ими допускалось при предложенном дележе (а точнее ровно столько же), а значит, не имея также и конфликта интересов в отношении оплаты дележа, они не откажутся от такого распределения блага с требованием дополнительных переговоров.

Случай 2.2. таблицы аналогичен случаю 2.1. Тогда здесь нет конфликта интересов в отношении способа дележа, так как каждый наследник предлагает одинаковый исход дележа. Реализовав дележ указанным в таблице образом (когда наследнику $H^{1}$ достается подблаго $W_{2}$, а наследнику $H^{2}$ - подблаго $W_{1}$ ), наследники $H^{1}$ и $H^{2}$ будут полагать, что в денежном выражении они получают, соответственно: $D_{01}^{1}=Ц_{01}^{1} / 2$ и $D_{01}^{2}=\bigsqcup_{01}^{2} / 2$.

\section{Случай 3.1. таблицы.}

Здесь имеется конфликт интересов в отношении способа дележа (когда наследник $H^{1}$ хочет, чтобы подблаго $W_{1}$ досталось ему, а подблаго $W_{2}$ - наследнику $H^{2}$, в то время как для наследника $H^{2}$ желательно наоборот; иными словами, для каждого наследника в первую очередь желаемо подблаго $W_{1}$, а о степени желательности подблага $W_{2}$ они ничего не сообщают).

Для разрешения конфликта предлагается выполнить тот дележ $\left(\delta_{1}, \delta_{2}\right)$, равный $(1,0)$ или $(0,1)$, который заявлен наследником $H^{2}$, готовым заплатить за указанную реализацию больше.

При этом такой наследник $H^{z}$ должен будет заплатить конкуренту $H^{\bar{z}}$ компромиссную или справедливую (в смысле равноправия сторон по отношению к распределяемому между ними благу) компенсацию, которая составит половину от указанного типа справедливой цены результата дележа, то есть величину $K_{\delta_{1} \delta_{2}}^{z}=\left(\frac{Ц_{10}^{1}+Ц_{01}^{2}}{2}\right) / 2=\frac{Ц_{10}^{1}+Ц_{01}^{2}}{4}$ у.д.е.

Кроме того заметим, что так как предложенный наследником $H^{z}$ дележ касался получения им только одного подблага, то за по- 
лучение конкурентом другого подблага компенсация самому наследнику $H^{z}$ от наследника $H^{\bar{z}}$ не предоставляется.

Тогда при реализации дележа указанным образом наследники $H^{1}$ и $H^{2}$ будут полагать, что в денежном выражении они получают, соответственно:

3.1.1. При $\bigsqcup_{10}^{1} / 2>Ц_{01}^{2} / 2 \geq \bigsqcup_{10}^{2} / 2$ (неравенство $\bigsqcup_{01}^{2} / 2 \geq Ц_{10}^{2} / 2$ добавлено в качестве уточнения о том, что если бы оно было неверно, то для наследника $H^{2}$ предпочтительнее был бы не дележ $(0,1)$, а дележ $(1,0))$ имеем:

$$
\begin{aligned}
D_{10}^{1}=Ц_{10}^{1}-\frac{Ц_{10}^{1}+Ц_{01}^{2}}{4}=\frac{3 Ц_{10}^{1}-Ц_{01}^{2}}{4} \geq 0 \\
\text { и } \quad D_{01}^{2}=D_{01}^{2}(1,0)=\frac{Ц_{10}^{1}+Ц_{01}^{2}}{4}+Ц_{10}^{2} \geq 0 .
\end{aligned}
$$

Покажем, что в этом случае каждый наследник получит не меньше, чем им допускалось при предложенном дележе, а значит при отсутствии учета «зависти» он не откажется от обсуждаемого распределения блага, требуя дополнительных переговоров. Действительно, согласно приведенным здесь неравенствам имеем:

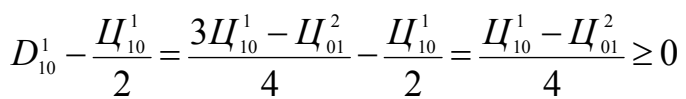

$$
\begin{aligned}
& \text { и } \quad D_{01}^{2}-\frac{Ц_{01}^{2}}{2}=\frac{Ц_{10}^{1}+Ц_{01}^{2}}{4}+Ц_{10}^{2}-\frac{Ц_{01}^{2}}{2}= \\
& =\frac{Ц_{10}^{1}-Ц_{01}^{2}}{4}+Ц_{10}^{2} \geq 0 \text {. }
\end{aligned}
$$

3.1.2. При $\bigsqcup_{01}^{2} / 2>Ц_{10}^{1} / 2 \geq Ц_{01}^{1} / 2$ имеем аналогичный результат:

$$
\begin{gathered}
D_{10}^{1}=\frac{Ц_{10}^{1}+\bigsqcup_{01}^{2}}{4}+Ц_{01}^{1} \geq 0 \\
\text { и } \quad D_{01}^{2}=Ц_{01}^{2}-\frac{Ц_{10}^{1}+\bigsqcup_{01}^{2}}{4}=\frac{-Ц_{10}^{1}+3 Ц_{01}^{2}}{4} \geq 0 .
\end{gathered}
$$

И в этом случае, как и в п. 3.1.1., доказывается, что каждый наследник получит не меньше, чем им допускалось при предложенном дележе, а значит при отсутствии учета «зависти» не откажется от обсуждаемого распределения блага, требуя дополнительных переговоров.

Случай 3.2. таблицы аналогичен случаю 3.1. Тогда здесь имеется конфликт инте- ресов (когда для каждого наследника в первую очередь желаемо подблаго $W_{2}$, а о степени желательности подблага $W_{1}$ они ничего не сообщают). Для разрешения конфликта предлагается выполнить тот дележ $\left(d_{1}, d_{2}\right)$, равный $(1,0)$ или $(0,1)$, который заявлен наследником $H^{z}$, готовым заплатить за указанную реализацию больше, выплачивающим в этом случае конкуренту компенсацию $K_{\delta_{1} \delta_{2}}^{z}=\frac{Ц_{01}^{1}+Ц_{10}^{2}}{4}$. А при реализации дележа указанным образом наследники $H^{1}$ и $H^{2}$ будут полагать, что в денежном выражении они получают, соответственно:

3.2.1. При $\bigsqcup_{01}^{1} / 2>\bigsqcup_{10}^{2} / 2 \geq \bigsqcup_{01}^{2} / 2$ имеем:

$$
\begin{gathered}
D_{01}^{1}=Ц_{01}^{1}-\frac{Ц_{01}^{1}+Ц_{10}^{2}}{4}=\frac{3 Ц_{01}^{1}-Ц_{10}^{2}}{4} \geq 0 \\
D_{10}^{2}=\frac{Ц_{01}^{1}+Ц_{10}^{2}}{4}+\coprod_{01}^{2} \geq 0 .
\end{gathered}
$$

3.2.2. При $\bigsqcup_{10}^{2} / 2>\bigsqcup_{01}^{1} / 2 \geq \bigsqcup_{10}^{1} / 2$ имеем:

$$
D_{01}^{1}=\frac{Ц_{01}^{1}+Ц_{10}^{2}}{4}+Ц_{10}^{1} \geq 0
$$

и $\quad D_{10}^{2}=Ц_{10}^{2}-\frac{Ц_{01}^{1}+Ц_{10}^{2}}{4}=\frac{-Ц_{01}^{1}+3 Ц_{10}^{2}}{4} \geq 0$.

\section{Случай 4.1. таблицы.}

Здесь имеется конфликт интересов с несовпадением мнений в отношении способа дележа (когда наследник $H^{1}$ хочет, чтобы и подблаго $W_{1}$, и подблаго $W_{2}$ досталось ему, а наследник $H^{2}$ хочет получить подблаго $W_{1}$ ).

Для разрешения этого конфликта предлагается выполнить тот дележ $\left(\delta_{1}, \delta_{2}\right)$, равный $(1,1)$ или $(0,1)$, который заявлен наследником $H^{z}$, готовым заплатить за указанную реализацию больше.

При этом такой наследник $H^{z}$ должен будет заплатить конкуренту $H^{\bar{z}}$ некоторую компенсацию $K_{\delta_{1} \delta_{2}}^{z}$. На данный момент дележа говорить о ее справедливости (в смысле равноправия сторон по отношению к распределяемому между ними благу) не представляется возможным, так как здесь наследники формулируют свои предложения в отношении неодинаковых или взаимодополняющих способах дележа, пока ничего дополнительно не сообщая друг другу. Уточним реализацию указанного способа дележа. 
4.1.1. При $Ц_{11}^{1} / 2>Ц_{01}^{2} / 2$ выполняется дележ $\left(d_{1}, d_{2}\right)=(1,1)$, и наследник $H^{1}$ выплачивает наследнику $H^{2}$ компенсацию в размере запрошенного им: $K_{11}^{1}=Ц_{01}^{2} / 2$. Тогда наследники $H^{1}$ и $H^{2}$ будут полагать, что в денежном выражении они получают, соответственно:

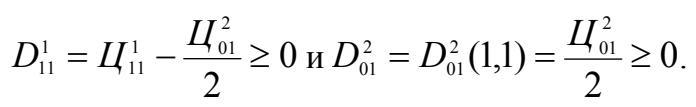

В этом случае каждый наследник получает не меньше, чем им допускалось при предложенном дележе:

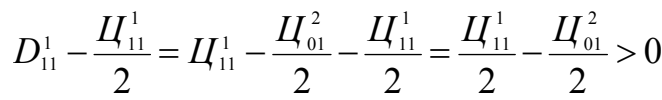

$$
\begin{aligned}
& \text { и } \quad D_{01}^{2}-\frac{Ц_{01}^{2}}{2}=\frac{Ц_{01}^{2}}{2}-\frac{Ц_{01}^{2}}{2}=0 \geq 0 \text {. }
\end{aligned}
$$

Следовательно, при отсутствии учета «зависти» никто из наследников не откажется от такого распределения блага с требованием дополнительных переговоров.

4.1.2. При $\bigsqcup_{01}^{2} / 2>Ц_{11}^{1} / 2 \geq \bigsqcup_{01}^{1} / 2$ (неравенство $\bigsqcup_{11}^{1} / 2 \geq Ц_{01}^{1} / 2$ добавлено в качестве уточнения о том, что если бы оно было неверно, то для наследника $H^{1}$ предпочтительнее был бы не дележ $(1,1)$, а дележ $(0,1))$ выполняется дележ $\left(\delta_{1}, \delta_{2}\right)=(0,1)$, и наследник $H^{2}$ выплачивает наследнику $H^{1}$ компенсацию в размере запрошенного им: $K_{01}^{2}=L_{11}^{1} / 2$. Тогда наследники $H^{1}$ и $H^{2}$ будут полагать, что в денежном выражении они получают, соответственно:

$D_{11}^{1}=D_{11}^{1}(0,1)=Ц_{01}^{1}+\frac{Ц_{11}^{1}}{2} \geq 0$ и $D_{01}^{2}=Ц_{01}^{2}-\frac{Ц_{11}^{1}}{2} \geq 0$.

В этом случае каждый наследник получает не меньше, чем им допускалось при предложенном дележе:

$$
D_{11}^{1}-\frac{Ц_{11}^{1}}{2}=\frac{Ц_{11}^{1}}{2}+Ц_{01}^{1}-\frac{Ц_{11}^{1}}{2}=Ц_{01}^{1} \geq 0
$$

и $D_{01}^{2}-\frac{Ц_{01}^{2}}{2}=Ц_{01}^{2}-\frac{Ц_{11}^{1}}{2}-\frac{Ц_{01}^{2}}{2}=\frac{Ц_{01}^{2}}{2}-\frac{Ц_{11}^{1}}{2}>0$.

Следовательно, и здесь при отсутствии учета «зависти» никто из наследников не откажется от такого распределения блага с требованием дополнительных переговоров.

Случай 4.2. таблицы аналогичен случаю 4.1. Тогда здесь имеется конфликт интересов с несовпадением мнений в отношении способа дележа (когда наследник $H^{1}$ хочет, чтобы и подблаго $W_{1}$, и подблаго $W_{2}$ досталось ему, а наследник $H^{2}$ хочет получить подблаго $W_{2}$ ).

Для разрешения этого конфликта предлагается выполнить тот дележ $\left(\delta_{1}, \delta_{2}\right)$, равный $(1,1)$ или $(1,0)$, который заявлен наследником $H^{z}$, готовым заплатить за указанную реализацию больше. При этом такой наследник $H^{z}$ должен будет заплатить конкуренту $H^{\bar{z}}$ некоторую компенсацию $K_{\delta, \delta}^{z}$. Точнее:

4.2.1. При $Ц_{11}^{1} / 2>Ц_{10}^{2} / 2$ выполняется дележ $\left(\delta_{1}, \delta_{2}\right)=(1,1)$, и наследник $H^{1}$ выплачивает наследнику $H^{2}$ компенсацию в размере запрошенного им: $K_{11}^{1}=L_{10}^{2} / 2$. Тогда наследники $H^{1}$ и $H^{2}$ будут полагать, что в денежном выражении они получают, соответственно:

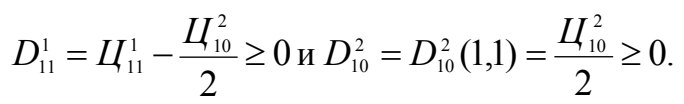

4.2.2. При $\bigsqcup_{10}^{2} / 2>\bigsqcup_{11}^{1} / 2 \geq \bigsqcup_{10}^{1} / 2$ выполняется дележ $\left(\delta_{1}, \delta_{2}\right)=(1,0)$, и наследник $H^{2}$ выплачивает наследнику $H^{1}$ компенсацию в размере запрошенного им: $K_{10}^{2}=Ц_{11}^{1} / 2$. Тогда наследники $H^{1}$ и $H^{2}$ будут полагать, что в денежном выражении они получают, соответственно:

и

$$
\begin{gathered}
D_{11}^{1}=D_{11}^{1}(1,0)=Ц_{10}^{1}+\frac{Ц_{11}^{1}}{2} \geq 0 \\
D_{10}^{2}=Ц_{10}^{2}-\frac{Ц_{11}^{1}}{2} \geq 0 .
\end{gathered}
$$

Случай 4.3. таблицы аналогичен случаю 4.1.

Здесь имеется конфликт интересов с несовпадением мнений в отношении способа дележа (когда наследник $H^{1}$ хочет получить подблаго $W_{1}$, а наследник $H^{2}$ хочет, чтобы и подблаго $W_{1}$, и подблаго $W_{2}$ досталось ему).

Для разрешения этого конфликта предлагается выполнить тот дележ $\left(\delta_{1}, \delta_{2}\right)$, равный $(1,0)$ или $(0,0)$, который заявлен наследником $H^{z}$, готовым заплатить за указанную реализацию больше. При этом такой наследник $H^{z}$ должен будет заплатить конкуренту $H^{\bar{z}}$ некоторую компенсацию $K_{\delta_{1}, \delta_{2}}^{z}$. Точнее:

4.3.1. При $\bigsqcup_{10}^{1} / 2>Ц_{00}^{2} / 2 \geq Ц_{10}^{2} / 2$ выполняется дележ $\left(\delta_{1}, \delta_{2}\right)=(1,0)$, и наследник $H^{1}$ выплачивает наследнику $H^{2}$ компенсацию в размере запрошенного им: $K_{10}^{1}=Ц_{00}^{2} / 2$. Тогда наследники $H^{1}$ и $H^{2}$ будут полагать, что в де- 
нежном выражении они получают, соответственно:

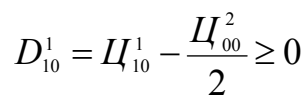

$$
\begin{aligned}
& \text { и } D_{00}^{2}=D_{00}^{2}(1,0)=\bigsqcup_{10}^{2}+\frac{Ц_{00}^{2}}{2} \geq 0 \text {. }
\end{aligned}
$$

4.3.2. При $\bigsqcup_{00}^{2} / 2>\bigsqcup_{10}^{1} / 2$ выполняется дележ $\left(\delta_{1}, \delta_{2}\right)=(0,0)$, и наследник $H^{2}$ выплачивает наследнику $H^{1}$ компенсацию в размере запрошенного им: $K_{00}^{2}=Ц_{10}^{1} / 2$. Тогда наследники $H^{1}$ и $H^{2}$ будут полагать, что в денежном выражении они получают, соответственно:

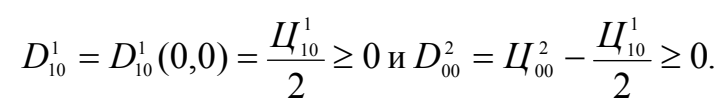

Случай 4.4. таблицы аналогичен случаю4.1.

Здесь имеется конфликт интересов с несовпадением мнений в отношении способа дележа (когда наследник $H^{1}$ хочет получить подблаго $W_{2}$, а наследник $H^{2}$ хочет, чтобы и подблаго $W_{1}$, и подблаго $W_{2}$ досталось ему).

Для разрешения этого конфликта предлагается выполнить тот дележ $\left(\delta_{1}, \delta_{2}\right)$, равный $(0,1)$ или $(0,0)$, который заявлен наследником $H^{z}$, готовым заплатить за указанную реализацию больше. При этом такой наследник $H^{z}$ должен будет заплатить конкуренту $H^{\bar{z}}$ некоторую компенсацию $K_{\delta_{1} \delta_{2}}^{z}$. Точнее:

4.4.1. При $\bigsqcup_{01}^{1} / 2>Ц_{00}^{2} / 2 \geq \bigsqcup_{01}^{2} / 2$ выполняется дележ $\left(\delta_{1}, \delta_{2}\right)=(0,1)$, и наследник $H^{1}$ выплачивает наследнику $H^{2}$ компенсацию в размере запрошенного им: $K_{01}^{1}=\bigsqcup_{00}^{2} / 2$. Тогда наследники $H^{1}$ и $H^{2}$ будут полагать, что в денежном выражении они получают, соответственно:

$$
\begin{gathered}
D_{01}^{1}=Ц_{01}^{1}-\frac{\bigsqcup_{00}^{2}}{2} \geq 0 \\
\text { и } \quad D_{00}^{2}=D_{00}^{2}(0,1)=Ц_{01}^{2}+\frac{Ц_{00}^{2}}{2} \geq 0 .
\end{gathered}
$$

4.4.2. При $\bigsqcup_{00}^{2} / 2>Ц_{01}^{1} / 2$ выполняется дележ $\left(\delta_{1}, \delta_{2}\right)=(0,0)$, и наследник $H^{2}$ выплачивает наследнику $H^{1}$ компенсацию в размере запрошенного им: $K_{00}^{2}=\bigsqcup_{01}^{1} / 2$. Тогда наследники $H^{1}$ и $H^{2}$ будут полагать, что в денежном выражении они получают, соответственно:

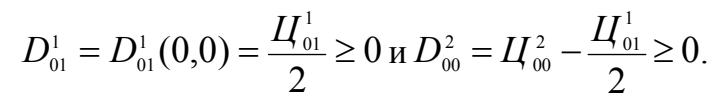

\section{Случай 5.1. таблицы.}

Здесь имеется конфликт интересов с несовпадением мнений в отношении способа дележа (когда наследник $H^{1}$ хочет получить подблаго $W_{2}$, а наследник $H^{2}$ готов за предоставление ему некоторой суммы отказаться и от подблага $W_{1}$, и от подблага $W_{2}$ ). Тогда возможны следующие ситуации.

5.1.1. При $\bigsqcup_{01}^{1} / 2>-\bigsqcup_{11}^{2} / 2 \geq \bigsqcup_{01}^{2} / 2$ выполняется дележ $\left(\delta_{1}, \delta_{2}\right)=(0,1)$, и наследник $H^{1}$ выплачивает наследнику $H^{2}$ компенсацию в размере запрошенного им: $K_{01}^{1}=-Ц_{11}^{2} / 2$. Тогда наследники $H^{1}$ и $H^{2}$ будут полагать, что в денежном выражении они получают, соответственно:

$$
\begin{gathered}
D_{01}^{1}=\bigsqcup_{01}^{1}-\frac{-\bigsqcup_{11}^{2}}{2} \geq 0 \\
D_{11}^{2}=D_{11}^{2}(0,1)=\bigsqcup_{01}^{2}+\frac{-\bigsqcup_{11}^{2}}{2} \geq 0 .
\end{gathered}
$$

В этом случае каждый наследник получает не меньше, чем им допускалось при предложенном дележе:

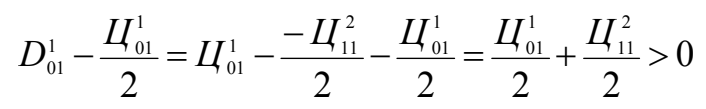

$$
\begin{aligned}
& \text { и } D_{11}^{2}-\frac{-\bigsqcup_{11}^{2}}{2}=\bigsqcup_{01}^{2}+\frac{-\bigsqcup_{11}^{2}}{2}-\frac{-\bigsqcup_{11}^{2}}{2}=\bigsqcup_{01}^{2} \geq 0 \text {. }
\end{aligned}
$$

Следовательно, при отсутствии учета «зависти» никто из наследников не откажется от такого распределения блага с требованием дополнительных переговоров.

5.1.2. При $-\bigsqcup_{11}^{2} / 2>\bigsqcup_{01}^{1} / 2$ реализовать заявленный наследником $H^{2}$ дележ не получится, так как наследник $H^{1}$ не станет в этом случае выплачивать ему требуемую компенсацию (будучи готовым платить не больше $\left.\bigsqcup_{01}^{1} / 2\right)$, а наследник $H^{2}$ не согласится на меньшее, чем $-\bigsqcup_{11}^{2} / 2$. То есть стороны конфликта не договорятся и придется осуществлять новый этап переговоров с дисконтированием цен.

Однако если, как и в п. 5.1.1., реализовать дележ $(0,1)$ с некоторой компенсацией $K_{01}^{1}$ от наследника $H^{1}$ к наследнику $H^{2}$, то наследники $H^{1}$ и $H^{2}$ будут полагать, что в денежном выражении они получают, соответственно:

$$
\begin{gathered}
\left.D_{01}^{1}=\bigsqcup_{01}^{1}-K_{01}^{1} \geq 0 \text { у.д.е. (то есть } K_{01}^{1} \leq Ц_{01}^{1}\right) \\
\text { и } \quad D_{11}^{2}=D_{11}^{2}(0,1)=\bigsqcup_{01}^{2}+K_{01}^{1} \geq 0 \text { у.д.е. }
\end{gathered}
$$


Выясним, когда наследнику $H^{1}$ дележ $(0,1)$ выгоднее, нежели отказ от него и организация нового этапа переговоров с ценами, дисконтированными посредством коэффициента $\delta$. Для этого (с учетом того, что в рассматриваемом случае $\left.L_{01}^{1}=\max \left\{L_{00}^{1}, L_{01}^{1}, L_{10}^{1}, L_{11}^{1}\right\}\right)$ должно быть выполнено неравенство $D_{01}^{1}=Ц_{01}^{1}-K_{01}^{1} \geq \delta \cdot Ц_{01}^{1}$, откуда $K_{01}^{1} \leq Ц_{01}^{1} \cdot(1-\delta) \geq 0$.

Кроме того, наследнику $H^{1}$ нужно получить в денежном выражении не меньше предлагаемого им за дележ $(0,1)$, то есть также должно быть выполнено

$D_{01}^{1}-\frac{Ц_{01}^{1}}{2}=Ц_{01}^{1}-K_{01}^{1}-\frac{Ц_{01}^{1}}{2}=\frac{Ц_{01}^{1}}{2}-K_{01}^{1} \geq 0$,

откуда $K_{01}^{1} \leq \frac{L_{01}^{1}}{2}$. В итоге здесь дележ $(0,1)$ выгоднее наследнику $H^{1}$, чем организация нового этапа переговоров, когда

$K_{01}^{1} \leq \min \left\{L_{01}^{1} \cdot(1-\delta) ; \frac{L_{01}^{1}}{2}\right\}$.

Выясним при отсутствии учета «зависти», когда наследнику $H^{2}$ дележ $(0,1)$ выгоднее, нежели отказ от него и организация нового этапа переговоров с ценами, дисконтированными посредством коэффициента $\delta$. Для этого (не забывая, что в рассматриваемом случае $\left.-Ц_{11}^{2}=\max \left\{Ц_{00}^{2}, Ц_{01}^{2}, Ц_{10}^{2},-Ц_{11}^{2}\right\}\right)$ должно быть выполнено неравенство

$D_{11}^{2}=Ц_{01}^{2}+K_{01}^{1} \geq \delta \cdot\left(-Ц_{11}^{2}\right)$, откуда $K_{01}^{1} \geq-\delta \cdot Ц_{11}^{2}-Ц_{01}^{2}$.

Кроме того, наследнику $H^{2}$ нужно получить в денежном выражении неменьше предлагаемого им за дележ $(0,1)$, то есть также должно быть выполнено $D_{11}^{2}-\frac{-Ц_{11}^{1}}{2}=Ц_{01}^{2}+K_{01}^{1}-\frac{-Ц_{11}^{1}}{2} \geq 0$, откуда $K_{01}^{1} \geq \frac{-Ц_{11}^{1}}{2}-Ц_{01}^{2}$. В итоге здесь дележ $(0,1)$ выгоднее наследнику $H^{1}$, чем организация нового этапа переговоров, когда

$K_{01}^{1} \geq \max \left\{-\delta \cdot Ц_{11}^{2}-Ц_{01}^{2} ; \frac{-Ц_{11}^{1}}{2}-Ц_{01}^{2}\right\}$.

Тогда (с учетом $\left.K_{01}^{1} \leq \min \left\{L_{01}^{1} \cdot(1-\delta) ; \frac{L_{01}^{1}}{2}\right\}\right)$ дележ $(0,1)$ реализовать следует, причем с минимальной по требованию наследника $H^{1}$ компенсацией $K_{01}^{1}=\max \left\{0 ;-\delta \cdot Ц_{11}^{2}-Ц_{01}^{2} ; \frac{-Ц_{01}^{1}}{2}-Ц_{01}^{2}\right\}$ (выясняемой в ходе дополнительного обсуждения ее величины, заранее неизвестной, поскольку наследнику $H^{1}$ наследником $H^{2}$ вначале сообщается лишь значение $\bigsqcup_{11}^{2} / 2$, но не $\bigsqcup_{01}^{2} / 2$ ), если $M=\left[\max \left\{0 ;-\delta \cdot Ц_{11}^{2}-Ц_{01}^{2} ; \frac{-Ц_{11}^{1}}{2}-Ц_{01}^{2}\right\}\right.$; $\left.\min \left\{Ц_{01}^{1} \cdot(1-\delta) ; \frac{Ц_{01}^{1}}{2}\right\}\right] \neq \varnothing$. В противном случае (при $M=\varnothing$ ) новый этап переговоров по способу дележа неизбежен.

Случай 5.2. таблицы аналогичен случаю 5.1.

Здесь имеется конфликт интересов с несовпадением мнений в отношении способа дележа (когда наследник $H^{1}$ хочет получить подблаго $W_{1}$, а наследник $H^{2}$ готов за предоставление ему некоторой суммы отказаться и от подблага $W_{1}$, и от подблага $W_{2}$ ). Тогда возможны следующие ситуации и решения в ходе них.

5.2.1. При $\bigsqcup_{10}^{1} / 2>-Ц_{11}^{2} / 2 \geq Ц_{10}^{2} / 2$ выполняется дележ $\left(\delta_{1}, \delta_{2}\right)=(1,0)$, и наследник $H^{1}$ выплачивает наследнику $H^{2}$ компенсацию в размере запрошенного им: $K_{10}^{1}=-Ц_{11}^{2} / 2$. Тогда наследники $H^{1}$ и $H^{2}$ будут полагать, что в денежном выражении они получают, соответственно:

и

$$
D_{10}^{1}=Ц_{10}^{1}-\frac{-Ц_{11}^{2}}{2} \geq 0
$$$$
D_{11}^{2}=D_{11}^{2}(1,0)=L_{10}^{2}+\frac{-Ц_{11}^{2}}{2} \geq 0 .
$$

5.2.2. При $-Ц_{11}^{2} / 2>Ц_{10}^{1} / 2$ выполняется дележ $(1,0) \quad$ с компенсацией $K_{10}^{1}=\max \left\{0 ;-\delta \cdot L_{11}^{2}-L_{10}^{2} ; \frac{-Ц_{11}^{2}}{2}-L_{10}^{2}\right\}$ от наследника $H^{1}$ к наследнику $H^{2}$ в случае, когда $M=\left[\max \left\{0 ;-\delta \cdot Ц_{11}^{2}-Ц_{10}^{2} ; \frac{-Ц_{11}^{2}}{2}-Ц_{10}^{2}\right\} ;\right.$ $\left.\min \left\{L_{10}^{1} \cdot(1-\delta) ; \frac{L_{10}^{1}}{2}\right\}\right] \neq \varnothing$ и нет учета «зависти». И тогда наследники $H^{1}$ и $H^{2}$ будут полагать, что в денежном выражении они получают, соответственно:

$$
D_{10}^{1}=Ц_{10}^{1}-K_{10}^{1} \geq 0
$$$$
\text { и } \quad D_{11}^{2}=D_{11}^{2}(1,0)=Ц_{10}^{2}+K_{10}^{1} \geq 0 \text {. }
$$

Если же $M=\varnothing$, то хотя бы одному наследнику выгоднее отказаться от таких условий и тогда организуется новый этап переговоров по способу дележа.

Случай 5.3. таблицы аналогичен случаю 5.1.

Здесь имеется конфликт интересов с несовпадением мнений в отношении способа дележа (когда наследник $H^{2}$ хочет получить под- 
благо $W_{2}$, а наследник $H^{1}$ готов за предоставление ему некоторой суммы отказаться и от подблага $W_{1}$, и от подблага $W_{2}$ ). Тогда возможны следующие ситуации и решения в ходе них.

5.3.1. При $-Ц_{00}^{1} / 2>\bigsqcup_{10}^{2} / 2$ выполняется дележ $(1,0) \quad$ с $\quad$ компенсацией $K_{10}^{2}=\max \left\{0 ;-\delta \cdot Ц_{00}^{1}-Ц_{10}^{1} ; \frac{-Ц_{00}^{1}}{2}-Ц_{10}^{1}\right\}$ от наследника $H^{2}$ к наследнику $H^{1}$ в случае, когда $M=\left[\max \left\{0 ;-\delta \cdot Ц_{00}^{1}-L_{10}^{1} ; \frac{-L_{00}^{1}}{2}-L_{10}^{1}\right\} ;\right.$ $\left.\min \left\{L_{10}^{2} \cdot(1-\delta) ; \frac{L_{10}^{2}}{2}\right\}\right] \neq \varnothing$ и нет учета «зависти». И тогда наследники $H^{1}$ и $H^{2}$ будут полагать, что в денежном выражении они получают, соответственно:

$$
D_{00}^{1}=D_{00}^{1}(1,0)=L_{10}^{1}+K_{10}^{2} \geq 0
$$

и

$$
D_{10}^{2}=Ц_{10}^{2}-K_{10}^{2} \geq 0 .
$$

Если же $M=\varnothing$, то хотя бы одному наследнику выгоднее отказаться от таких условий, и тогда организуется новый этап переговоров по способу дележа.

5.3.2. При $\bigsqcup_{10}^{2} / 2 \geq-Ц_{00}^{1} / 2 \geq \bigsqcup_{10}^{1} / 2$ выполняется дележ $\left(\delta_{1}, \delta_{2}\right)=(1,0)$, и наследник $H^{2}$ выплачивает наследнику $H^{1}$ компенсацию в размере запрошенного им: $K_{10}^{2}=-Ц_{00}^{1} / 2$. Тогда наследники $H^{1}$ и $H^{2}$ будут полагать, что в денежном выражении они получают, соответственно:

$$
\begin{aligned}
D_{00}^{1}= & D_{00}^{1}(1,0)=Ц_{10}^{1}+\frac{-Ц_{00}^{1}}{2} \geq 0 \\
\text { и } & D_{10}^{2}=Ц_{10}^{2}-\frac{-Ц_{00}^{1}}{2} \geq 0 .
\end{aligned}
$$

Случай 5.4. таблицы аналогичен случаю 5.1.

Здесь имеется конфликт интересов с несовпадением мнений в отношении способа дележа (когда наследник $H^{1}$ готов за предоставление ему некоторой суммы отказаться и от подблага $W_{1}$, и от подблага $W_{2}$, а наследник $H^{2}$ хочет получить подблаго $\left.W_{1}\right)$. Тогда возможны следующие ситуации и решения в ходе них.

5.4.1. При $-Ц_{00}^{1} / 2>Ц_{01}^{2} / 2$ выполняется дележ $(0,1) \quad$ с $\quad$ компенсацией $K_{01}^{2}=\max \left\{0 ;-\delta \cdot Ц_{00}^{1}-Ц_{01}^{1} ; \frac{-Ц_{00}^{1}}{2}-Ц_{01}^{1}\right\}$ от наследника $H^{2}$ к наследнику $H^{1}$ в случае, когда $M=\left[\max \left\{0 ;-\delta \cdot L_{00}^{1}-L_{01}^{1} ; \frac{-Ц_{00}^{1}}{2}-Ц_{01}^{1}\right\} ;\right.$ $\left.\min \left\{Ц_{01}^{2} \cdot(1-\delta) ; \frac{Ц_{01}^{2}}{2}\right\}\right] \neq \varnothing$ и нет учета «зависти». И тогда наследники $H^{1}$ и $H^{2}$ будут полагать, что в денежном выражении они получают, соответственно:

и

$$
\begin{gathered}
D_{00}^{1}=D_{00}^{1}(0,1)=L_{01}^{1}+K_{01}^{2} \geq 0 \\
D_{01}^{2}=Ц_{01}^{2}-K_{01}^{2} \geq 0 .
\end{gathered}
$$

Если же $M=\varnothing$, то хотя бы одному наследнику выгоднее отказаться от таких условий, и тогда организуется новый этап переговоров по способу дележа.

5.4.2. При $\bigsqcup_{01}^{2} / 2 \geq-Ц_{00}^{1} / 2 \geq \bigsqcup_{01}^{1} / 2$ выполняется дележ $\left(\delta_{1}, \delta_{2}\right)=(0,1)$, и наследник $H^{2}$ выплачивает наследнику $H^{1}$ компенсацию в размере запрошенного им: $K_{01}^{2}=-Ц_{00}^{1} / 2$. Тогда наследники $H^{1}$ и $H^{2}$ будут полагать, что в денежном выражении они получают, соответственно:

и

$$
\begin{gathered}
D_{00}^{1}=D_{00}^{1}(0,1)=Ц_{01}^{1}+\frac{-Ц_{00}^{1}}{2} \geq 0 \\
D_{01}^{2}=Ц_{01}^{2}-\frac{-Ц_{00}^{1}}{2} \geq 0 .
\end{gathered}
$$

\section{Случай 6 таблицы.}

Здесь имеется конфликт интересов с несовпадением мнений в отношении способа дележа (когда каждый наследник $H^{1}$ и $H^{2}$ хочет, чтобы и подблаго $W_{1}$, и подблаго $W_{2}$ досталось ему).

Для разрешения этого конфликта предлагается выполнить тот дележ $\left(\delta_{1}, \delta_{2}\right)$, равный $(1,1)$ или $(0,0)$, который заявлен наследником $H^{z}$, готовым заплатить за указанную реализацию больше.

При этом такой наследник $H^{z}$ должен будет заплатить конкуренту $H^{\bar{z}}$ компромиссную или справедливую (в смысле равноправия сторон по отношению к распределяемому между ними благу) компенсацию, которая составит половину от указанного типа справедливой цены результата дележа, то есть величину $K_{\delta_{1} \delta_{2}}^{z}=\left(\frac{Ц_{11}^{1}+Ц_{00}^{2}}{2}\right) / 2=\frac{Ц_{11}^{1}+Ц_{00}^{2}}{4}$ у.д.е.

Тогда при реализации дележа указанным образом наследники $H^{1}$ и $H^{2}$ будут полагать, что в денежном выражении они получают, соответственно:

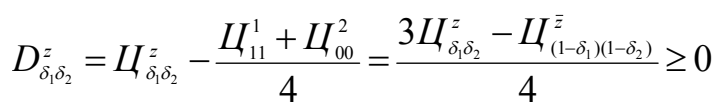




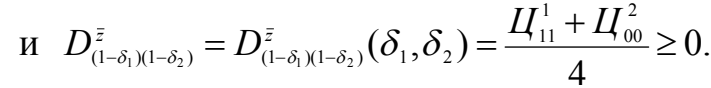

Покажем, что в этом случае каждый наследник получит не меньше, чем им допускалось при предложенном дележе, а значит он не откажется от такого распределения блага с требованием дополнительных переговоров. Действительно, согласно приведенным здесь неравенствам имеем:

$$
\begin{aligned}
& D_{\delta_{1} \delta_{2}}^{z}-\frac{\bigsqcup_{\delta_{1} \delta_{2}}^{z}}{2}=\frac{3 Ц_{\delta_{1} \delta_{2}}^{z}-Ц_{\left(1-\delta_{1}\right)\left(1-\delta_{2}\right)}^{\bar{z}}}{4}-\frac{\bigsqcup_{\delta_{1} \delta_{2}}^{z}}{2}= \\
& =\frac{\bigsqcup_{\delta_{1} \delta_{2}}^{z}-Ц_{\left(1-\delta_{1}\right)\left(1-\delta_{2}\right)}^{\bar{z}} \geq 0}{4} \geq \\
& \text { и } D_{\left(1-\delta_{1}\right)\left(1-\delta_{2}\right)}^{\bar{z}}-\frac{Ц_{\left(1-\delta_{1}\right)\left(1-\delta_{2}\right)}^{\bar{z}}}{2}=\frac{Ц_{10}^{1}+Ц_{01}^{2}}{4}-\frac{Ц_{\left(1-\delta_{1}\right)\left(1-\delta_{2}\right)}^{\bar{z}}}{2}=
\end{aligned}
$$

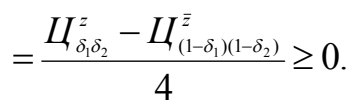

\section{Случай 7 таблицьь.}

Здесь имеется конфликт интересов с несовпадением мнений в отношении способа дележа (когда каждый наследник $H^{1}$ и $H^{2}$ готов за предоставление ему некоторой суммы отказаться и от подблага $W_{1}$, и от подблага $W_{2}$ ).

Для разрешения этого конфликта предлагается выполнить тот дележ $\left(\delta_{1}, \delta_{2}\right)$, равный $(0,0)$ или $(1,1)$, который заявлен наследником $H^{z}$, согласным на получение меньшей суммы за отказ от получения наследства (а значит, у которого предложенная отрицательная цена $Ц_{\delta_{1} \delta_{2}}^{z} / 2$ больше или, говоря иначе, у кого $\left.-\bigsqcup_{\delta_{1} \delta_{2}}^{z} / 2<-Ц_{\left(1-\delta_{1}\right)\left(1-\delta_{2}\right)}^{\bar{z}} / 2\right) . \quad$ Соответственно наследник $H^{z}$ не получит наследство, но получит за него от конкурента $H^{\bar{z}}$ компромиссную или справедливую (в смысле равноправия сторон по отношению к распределяемому между ними благу) компенсацию, которая составит половину от указанного типа справедливой цены результата дележа, то есть величину

$$
K_{\delta_{1} \delta_{2}}^{\bar{z}}=\left(\frac{-\bigsqcup_{00}^{1}-Ц_{11}^{2}}{2}\right) / 2=\frac{-\bigsqcup_{00}^{1}-Ц_{11}^{2}}{4} \text { у.д.е. }
$$

Тогда при реализации дележа указанным î áđàçi ̀̀ í àñëåäí èêè $H^{1}$ и $H^{2}$ будут полагать, что в денежном выражении они получают, соответственно:

$$
D_{\delta_{1} \delta_{2}}^{z}=\frac{-Ц_{00}^{1}-Ц_{11}^{2}}{4} \geq 0
$$

и $\quad D_{\left(1-\delta_{1}\right)\left(1-\delta_{2}\right)}^{\bar{z}}=D_{\left(1-\delta_{1}\right)\left(1-\delta_{2}\right)}^{\bar{z}}\left(\delta_{1}, \delta_{2}\right)=$

$=-\bigsqcup_{\left(1-\delta_{1}\right)\left(1-\delta_{2}\right)}^{\overline{2}}-\frac{-Ц_{11}^{1}-Ц_{00}^{2}}{4}=\frac{-3 Ц_{\left(1-\delta_{1}\right)\left(1-\delta_{2}\right)}^{\bar{z}}+\bigsqcup_{\delta_{1} \delta_{2}}^{z}}{4} \geq 0$.

Покажем, что в этом случае каждый наследник получит не меньше, чем им допускалось при предложенном дележе, а значит он не откажется от такого распределения блага с требованием дополнительных переговоров. Действительно, согласно приведенным здесь неравенствам имеем:

$$
\begin{gathered}
D_{\delta_{1} \delta_{2}}^{z}-\frac{-\bigsqcup_{\delta_{1} \delta_{2}}^{z}}{2}=\frac{-Ц_{00}^{1}-\bigsqcup_{11}^{2}}{4}-\frac{-\bigsqcup_{\delta_{1} \delta_{2}}^{z}}{2}= \\
=\frac{-\bigsqcup_{\left(1-\delta_{1}\right)\left(1-\delta_{2}\right)}^{\bar{z}}+\bigsqcup_{\delta_{1} \delta_{2}}^{z}}{4} \geq 0 \\
=\frac{-3 \bigsqcup_{\left(1-\delta_{1}\right)\left(1-\delta_{2}\right)}^{\bar{z}}+\bigsqcup_{\delta_{1} \delta_{2}}^{z}-\frac{-\bigsqcup_{\left(1-\delta_{1}\right)\left(1-\delta_{2}\right)}^{\bar{z}}}{4}=}{2} \\
=\frac{-\bigsqcup_{\left(1-\delta_{1}\right)\left(1-\delta_{2}\right)}^{\bar{z}}+\bigsqcup_{\delta_{1} \delta_{2}}^{z} \geq 0 .}{4}=0
\end{gathered}
$$$$
\text { и }
$$

\section{Заключение}

Несмотря на естественное предположение об относительно частом возникновении необходимости использования многоэтапных переговоров в условиях рассмотренной процедуры дележа, в рамках которой наследники сообщают друг другу не полные, а частичные сведения о системах своих предпочтений, в настоящей работе выяснилось, что в большинстве случаев без этих переговоров можно обойтись в ситуациях, когда понятие «зависть» не учитывается. А принятие во внимание указанного обстоятельства есть очередной шаг в изысканиях по теме.

Разрабатываемый в статье подход, берущий начало в публикации [3], демонстрирует еще одно возможное направление исследования, отличное от известных и развиваемых на основе трудов [1; 5]. Он позволяет производить анализ и принимать решения в условиях неполной информации.

Перечисленные во введении примеры применения изложенных в статье результатов демонстрируют типичные ситуации, характерные для человеческого общества и требующие их мирного разрешения, чему может способствовать данная работа. 


\section{СПИСОК ЛИТЕРАТУРЫ}

1. Брамс, С. Д. Делим по справедливости, или гарантия выигрыша каждому / С. Д. Брамс, А. Д. Тейлор.-М. : СИНТЕГ, 2002.-196 с.

2. Егоров, В. В. Немонотонность и конфликты при распределении блага с двумя неделимыми составляющими / В. В. Егоров // Экономика и менеджмент в условиях глобальной конкуренции: проблемы и перспективы : тр. науч.-практ. конф. с междунар. участием / под ред. д-ра экон. наук, проф. А. В. Бабкина. - СПб. : Изд-во Политехн. ун-та, 2016. - C. 39-48.

3. Егоров, В. В. Об одной модификации способа дележа неделимого наследства / В. В. Егоров // Анализ, моделирование и прогнозирование экономических процессов: материалы II Междунар. науч.-практ. интернет-конф. / под ред. Л. Ю. Богачковой, В. В. Давниса. - Воронеж : Изд-во ЦНТИ, 2010. - С. 127-132. - Электрон. текстовые дан. - Режим доступа: http://volsu.ru/forum/forum36/topic137/.Загл. с экрана.

4. Егоров, В. В. Об учете зависти в ходе распределения компенсаций при дележе неделимого блага между несколькими лицами / В. В. Егоров // Анализ, моделирование и прогнозирование экономических процессов : материалы V Междунар. науч.-практ. интернет-конф. / под ред. Л. Ю. Богачковой, В. В. Давниса. - Воронеж : Изд-во ЦНТИ, 2013. - С. 133-135. - Электрон. текстовые дан. - Режим доступа: http://volsu.ru/forum/forum76/topic486/. - Загл. с экрана.

5. Мазалов, В. В. Переговоры. Математическая теория / В. В. Мазалов, А. Э. Менчер, Ю. С. Токарева. - СПб. : Лань, 2012. - 304 с.

\section{REFERENCES}

1. Brams S.J., Taylor A.D. Delim po spravedlivosti, ili garantiya vyigrysha kazhdomu [The Win-Win Solution: Guaranteeing Fair Shares to
Everybody]. Transl. by Yu.M. Yanovskaya. Moscow, Sinteg Publ., 2002. 196 p.

2. Egorov V.V. Nemonotonnost i konflikty pri raspredelenii blaga $\mathrm{s}$ dvumya nedelimymi sostavlyayushchimi [Non-Monotonicity and Conflicts at Distribution of Benefits with Two Indivisible Components]. Babkin A.V., ed. Ekonomika i menedzhment v usloviyakh globalnoy konkurentsii: problemy i perspektivy. Trudy nauchno-prakticheskoy konferentsii s mezhdunarodnym uchastiem [Economy and Management in the Conditions of the Global Competitions: Problems and Prospects. Proceedings of Research and Practice Conference with International Participation]. Saint Petersburg, Izd-vo Politekhn. un-ta, 2016, pp. 39-48.

3. Egorov V.V. Ob odnoy modifikatsii sposoba delezha nedelimogo nasledstva [On a Modification of the Way of Indivisible Inheritance Division]. Bogachkova L.Yu., Davnis V.V., eds. Analiz, modelirovanie i prognozirovanie economicheskikh protsessov: materialy II Mezhdunarodnoy nauchnoprakticheskoy Internet-konferentsii [The Analysis, Modelling and Forecasting of Economic Processes: Proceedings of the $2^{\text {nd }}$ International Research and Practice Internet Conference]. Voronezh, Izd-vo TsNTI, 2010, pp. 127-132. URL: http://volsu.ru/forum/forum36/topic137/.

4. Egorov V.V. Ob uchete zavisti v khode raspredeleniya kompensatsiy pri delezhe nedelimogo blaga mezhdu neskolkimi litsami [On the Accounting of Envy at Distribution of Compensation in the Division of Indivisible Benefit among Several Persons]. Bogachkova L.Yu., Davnis V.V., eds. Analiz, modelirovanie i prognozirovanie economicheskikh protsessov: materialy II Mezhdunarodnoy nauchnoprakticheskoy Internet-konferentsii [The Analysis, Modelling and Forecasting of Economic Processes: Proceedings of the $2^{\text {nd }}$ International Research and Practice Internet Conference]. Voronezh, Izd-vo TsNTI, 2013, pp. 133-135. URL: http://volsu.ru/forum/forum76/topic486/.

5. Mazalov V. V., Mencher A. E., Tokareva Yu. S. Peregovory. Matematicheskaya teoriya [Negotiation. Mathematical Theory]. Saint Petersburg, Lan Publ., 2012. $304 \mathrm{p}$.

\section{Information about the Author}

Vladislav V. Egorov, Candidate of Sciences (Physics and Mathematics), Associate Professor, Department of Mathematical Methods and Informatics in Economics, Volgograd State University, Prosp. Universitetsky, 100, 400062 Volgograd, Russian Federation, yegoroff_vv@mail.ru, mmie@volsu.ru.

\section{Информация об авторе}

Владислав Валерьевич Егоров, кандидат физико-математических наук, доцент кафедры математических методов и информатики в экономике, Волгоградский государственный университет, просп. Университетский, 100, 400062 г. Волгоград, Российская Федерация, yegoroff_vv@mail.ru, mmie@volsu.ru. 